\title{
Odontoma Compuesto: Diagnóstico y Tratamiente Reporte de Casos \& Revisión de la Literatura
}

\author{
Compound Odontoma: Diagnosis and Treatment Therapeutic Measures. \\ Case Reports \& Literature Review
}

\author{
Gonzalo Martinovic Guzmán'; Bernardita Santorcuato Cubillos; \\ Juan Pablo Alister Herdener ${ }^{3}$; Carlos Plaza Álvarez ${ }^{4} \&$ Jerko Raffo Solari ${ }^{4}$
}

MARTINOVIC, G. G.; SANTORCUATO, C. B.; ALISTER, H. J. P.; PLAZA, Á. C. \& RAFFO, S. J. Odontoma compuesto: Diagnóstico y tratamiento. Reporte de casos \& revisión de la literatura. Int. J. Odontostomat., 11(4):425-430, 2017.

RESUMEN: Los odontomas son los tumores odontogénicos benignos más prevalentes. Estos están conformados por tejido dentario, epitelio odontogénico y tejido mesenquimático. Se dividen en dos subtipos: odontomas compuestos, que presentan tejidos dentarios normales, pero con una alteración en su conformación y tamaño, y odontomas complejos, que presentan tejidos dentarios bien formados, pero rodeados de tejido desorganizado. Se presentan tres casos de pacientes sexo femenino, quienes acuden para evaluación y tratamiento debido al hallazgo radiográfico de odontoma compuesto. En el caso $n^{0} 1$ se realizó la desinclusión del diente impactado, la exodoncia del diente remanente, la exéresis del odontoma compuesto, la instalación de un implante dental en el alveolo de la zona intervenida y, finalmente, el posicionamiento de injerto óseo. En el caso $n^{\circ} 2$ se realizó la exodoncia del diente temporal remanente y la exéreis del odontoma compuesto. Ambos casos evolucionaron de manera favorable sin complicaciones post operatorias. En el caso $n^{\circ} 3$ se realizó la fenestración del diente 18 y la exéreis del odontoma compuesto que retenía su erupción. Todos los casos evolucionaron de manera favorable sin complicaciones post operatorias. Dada la prevalencia de esta patología, es necesario un adecuado conocimiento sobre ella y sus características, para realizar un adecuado diagnóstico y tratamiento. Existen distintas medidas terapéuticas respecto a la rehabilitación de una zona edéntula como resultado de la exéresis de estos tumores. Esto depende principalmente de la edad del paciente. Al ser requeridas medidas rehabilitadoras, un punto importante a considerar es la posibilidad de realizar todos los procedimientos quirúrgicos en un solo tiempo operatorio.

PALABRAS CLAVE: odontoma compuesto, diagnóstico, tratamiento.

\section{INTRODUCCIÓN}

Los odontomas son un grupo de tumores odontogénicos benignos, compuestos por tejido dentario más epitelio odontogénico y tejido mesenquimático. Son definidos como hamartomas y son los tumores odontogénicos más prevalentes (Salgado \& Mesquita, 2013). Son más prevalentes durante la primera y segunda década de vida, y no presentan predilección por sexo (Núñez et al., 2016). Rara vez erupcionan y eventualmente pueden generar expansión ósea (Núñez et al.).

Existen dos subtipos: los odontomas compues- tos, caracterizados por tejido dentario normal, alterados en su conformación y tamaño, dando así origen a dentículos; y los odontomas complejos, caracterizados por tejido dentario bien formados pero rodeados de tejido desorganizado (Thistle Barba et al., 2016). Entre ambos, son más frecuentes los odontomas compuestos (Salgado \& Mesquita).

Los odontomas compuestos se localizan preferentemente en la zona maxilar anterior, y los odontomas complejos frecuentemente en la zona mandibular posterior (Hidalgo-Sánchez et al., 2008).

\footnotetext{
${ }^{1}$ Cirujano Maxilofacial, Hospital Militar de Santiago, Programa Magister en Ciencias Medicas Mención Cirugía, Facultad de Medicina, Universidad de La Frontera, Temuco, Chile.

${ }^{2}$ Hospital Militar de Santiago, Santiago, Chile.

${ }^{3}$ Cirujano Maxilofacial, PhD, Universidad de la Frontera, Temuco, Chile.

${ }^{4}$ Cirujano Maxilofacial, Hospital Militar de Santiago, Santiago, Chile.
} 
Generalmente su diagnóstico se debe a hallazgos radiográficos, ya que son tumores asintomáticos. Los signos más frecuentes son una erupción dentaria retardada y persistencia de dientes temporales (Salgado \& Mesquita).

Los odontomas compuestos se presentan radiográficamente como múltiples dentículos claramente distinguidos rodeados por un halo radiolúcido; y los odontomas complejos como una o múltiples masas radiopacas rodeadas por un halo radiolúcido (Angiero et al., 2014).

\section{MATERIAL Y MÉTODO}

El estudio fue conducido en el Hospital Militar de Santiago, Chile, durante el año 2016, Se incluyeron todos los sujetos con diagnóstico de odontoma compuesto que fueron intervenidos quirúrgicamente durante el período antes señalado. El tiempo de seguimiento fue 1 año en ambos sujetos. Todos los pacientes firmaron su consentimiento informado y su identidad se mantuvo en reserva. Se realizó una revisión bibliográfica (Manterola \& Astudillo, 2013) en los buscadores Pubmed y Epistemonikos, con el término Mesh "Odontoma".

\section{Reporte de casos}

Caso clínico $n^{\circ}$ 1: Paciente sexo femenino, 18 años de edad, consulta para evaluación por presencia de odontoma en zona diente 2.3, retención de diente 6.3 y diente 2.3 incluido (Fig. 1). Diagnóstico es realizado previamente como hallazgo radiográfico en relación a consulta por permanencia de diente 6.3. Se solicita Cone Beam maxilar, donde se aprecia diente 2.3 incluido en zona palatina y en relación a múltiples dentículos (cuatro) y permanencia de diente 6.3 con reabsorción radicular (Fig. 2). Diagnóstico odontoma compuesto en zona diente 2.3.
La paciente es sometida a anestesia general, donde se realiza, en primer lugar, la desinclusión palatina del diente 23. A continuación, se realiza la extracción del diente 63 y la exéresis del odontoma compuesto vía alveolar y labial, preservando el hueso alveolar lo mayor posible.

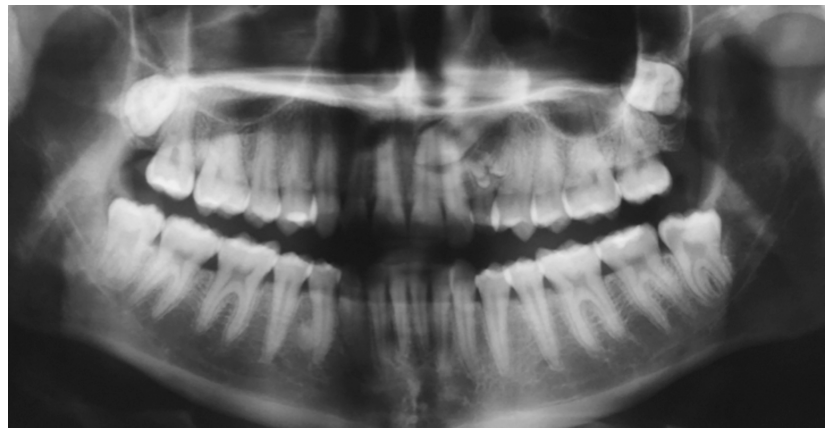

Fig.1. Radiografía panorámica. Se evidencian dentículos definidos de odontoma compuesto en relación a diente 23 , diente 23 incluido y permanencia diente 63.

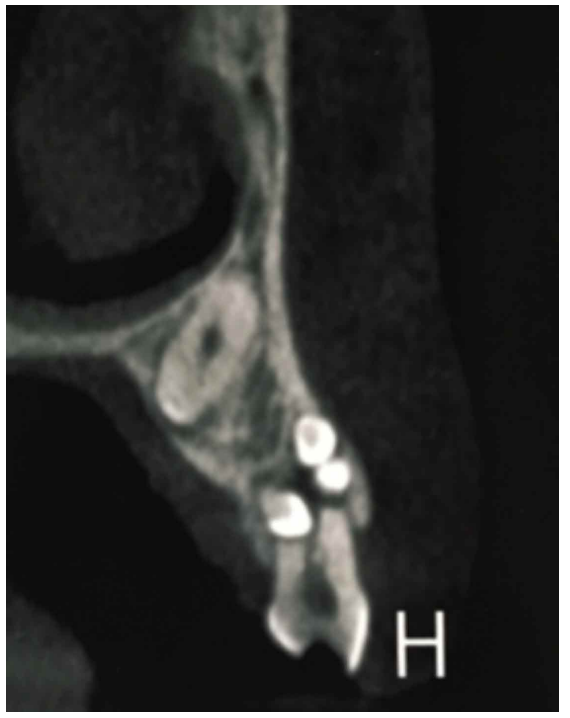

Fig. 2. Corte sagital CBCT. Diente 23 incluido con permanencia de diente 63 y presencia de tres dentículos definidos en relación al diente

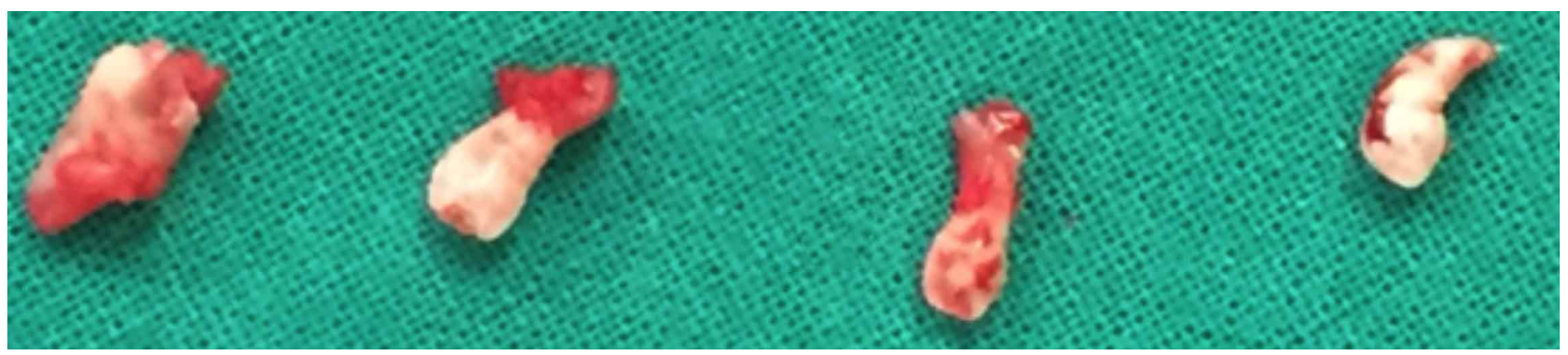

Fig. 3. Cuatro dentículos de odontoma compuesto luego de su exéresis. 
Una vez finalizada la exéresis de los cuatro dentículos (Fig. 3) que conformaban el odontoma compuesto, se realiza el protocolo de fresado y la instalación de un implante oseointegrado AlphaBio SPI 3,75 x 11,5 mm, más injerto óseo de 1 cc (Alpha Bio Graft) en zonas palatina, alveolar y labial en relación a implante zona diente 2.3 (Figs. 4 y 5 ).

El estudio de biopsia obtuvo como resultado el diagnóstico de odontoma compuesto.

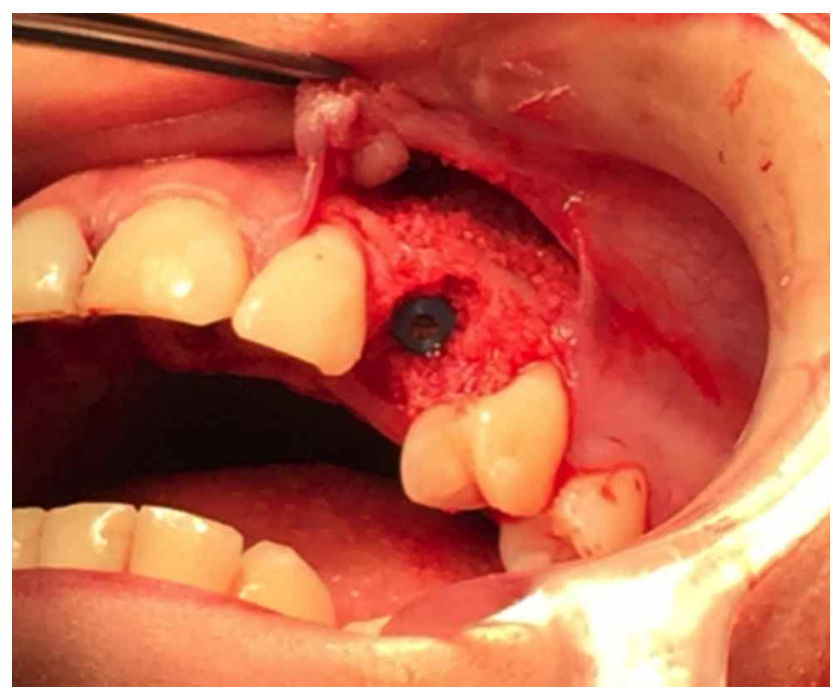

Fig. 4. Implante dental instalado en zona de diente 2.3 posicionado en conjunto con el injerto óseo (Alpha Bio Graft).

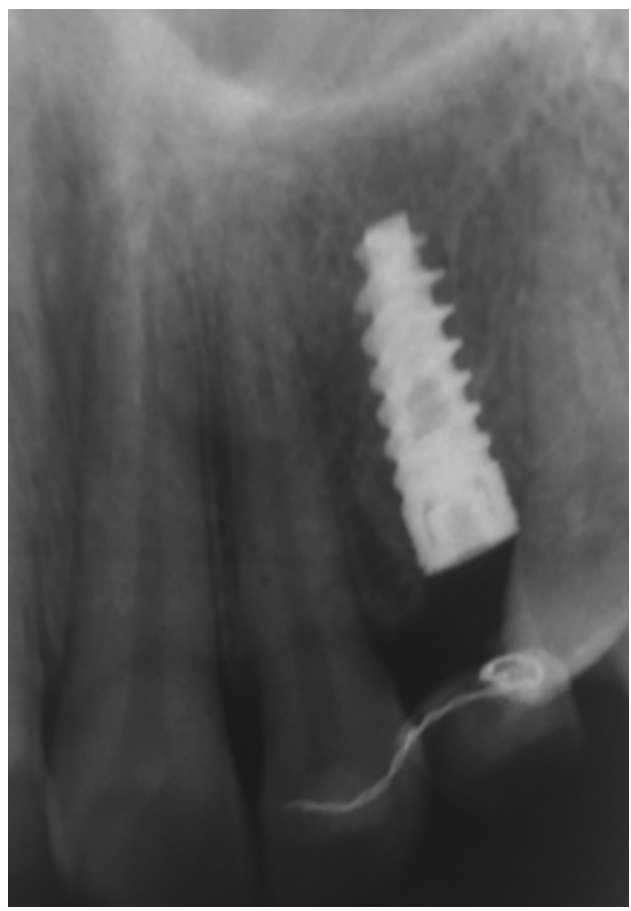

Fig. 5. Radiografía periapical. Implante dental instalado en zona diente 2.3.
Caso clínico $n^{\circ}$ 2: Paciente sexo femenino, 9 años de edad, consulta para evaluación por presencia de odontoma en zona diente 2.1, retención de diente 6.1 e inclusión del diente 2.1 (Fig. 6). Diagnóstico es realizado previamente como hallazgo radiográfico en relación a consulta por retraso de erupción de diente 2.1 y permanencia de diente 6.1. Se solicita Cone Beam maxilar, donde se aprecia diente 2.1 incluido retenido por múltiples dentículos en relación a su porción coronaria (Fig. 7). Diagnóstico odontoma compuesto en zona diente 2.1.

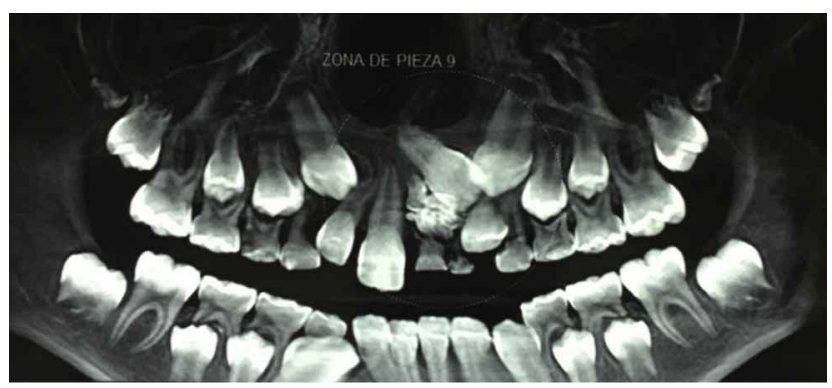

Fig. 6. CBCT. Diente 2.1 incluido. Presencia de odontoma compuesto en relación al diente, y permanencia diente 6.1 .

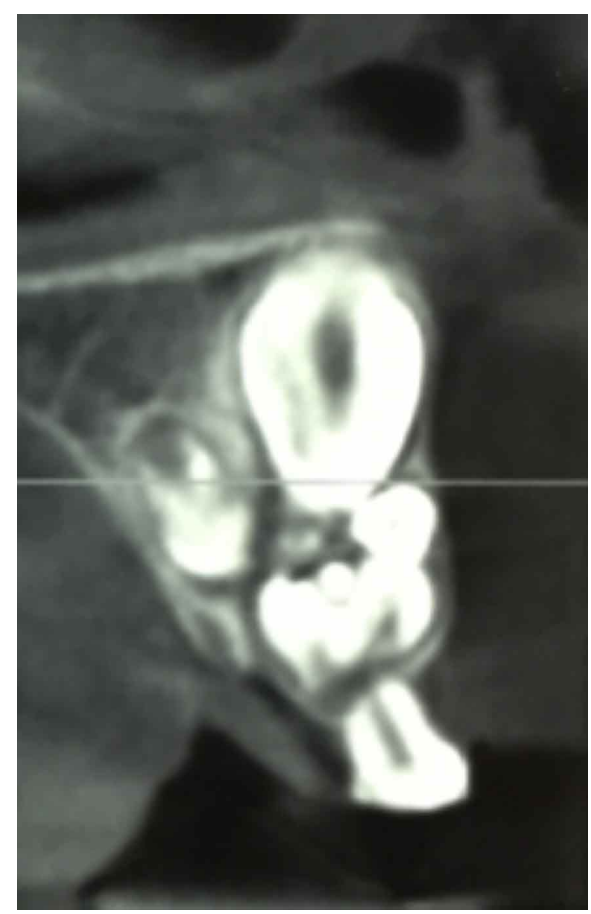

Fig. 7. Corte sagital CBCT. Diente 2.1 incluido, múltiples dentículos definidos en relación a éste, y permanencia diente 6.1.

Paciente es sometida a anestesia general en pabellón central, donde se realiza la extracción del diente 6.1 y la exéresis del odontoma compuesto (Fig. 8) y sus múltiples dentículos vía alveolar (Fig. 9). 


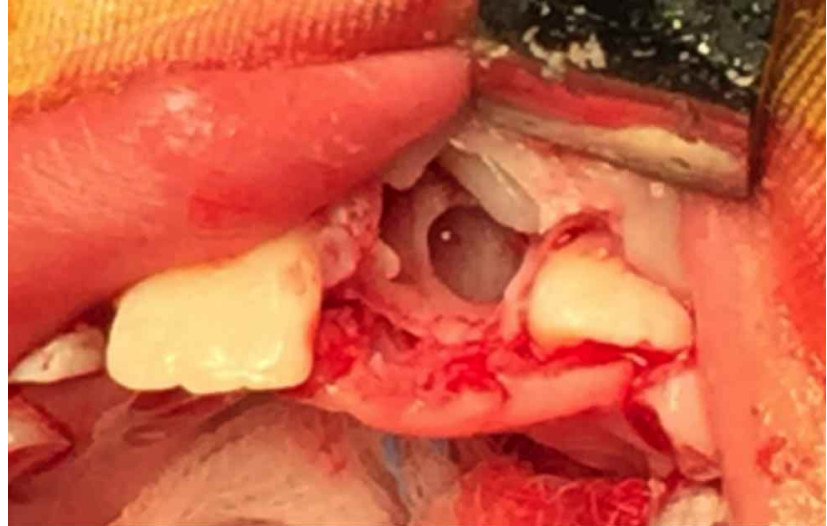

Fig. 8. Visión del alveolo después de la exodoncia y exéresis del odontoma compuesto.

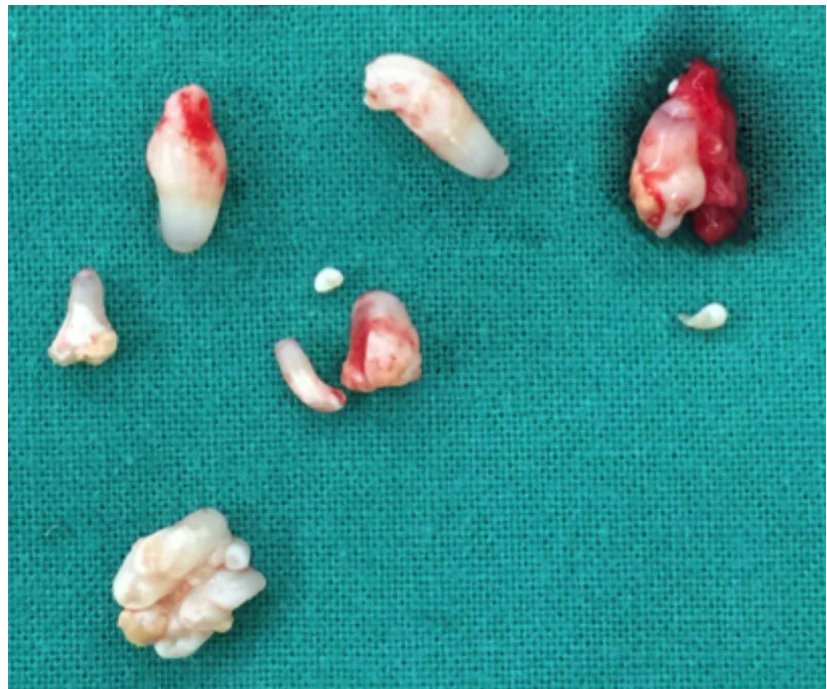

Fig. 9. Múltiples dentículos luego de la exéresis del odontoma compuesto.

\section{Caso clínico $n^{\circ} 3$}

Paciente sexo femenino, 9 años de edad, consulta para evaluación por presencia de odontoma en zona diente 1.1, con retención del diente indicado. El Diagnóstico es realizado previamente como hallazgo radiográfico en relación a consulta por retraso de erupción del diente 2.1. Se solicita Cone Beam maxilar, donde se aprecia diente 1.1 incluido y retenido por múltiples dentículos en relación a su porción coronaria (Fig.10). Diagnóstico odontoma compuesto en zona diente 1.1.

Paciente es sometida a anestesia general en pabellón central, donde se realiza la fenestración del diente 1.1 y la exéresis del odontoma compuesto y sus múltiples dentículos vía alveolar más la exodoncia de dientes temporales $5.3,6.3,7.3$ y 8.3 .
Al realizar la revisión de la literatura, en Pubmed fueron encontradas 3 revisiones sistemáticas, y 15 estudios clínicos. En Epistemonikos fue encontrada solo una revisión sistemática, coincidente con una de las revisiones sistemáticas de Pubmed. De aquellas revisiones sistemáticas encontradas en Pubmed, 2 fueron filtradas por título y abstract y solo una fue seleccionada; la misma revisión encontrada en Epistemonikos.

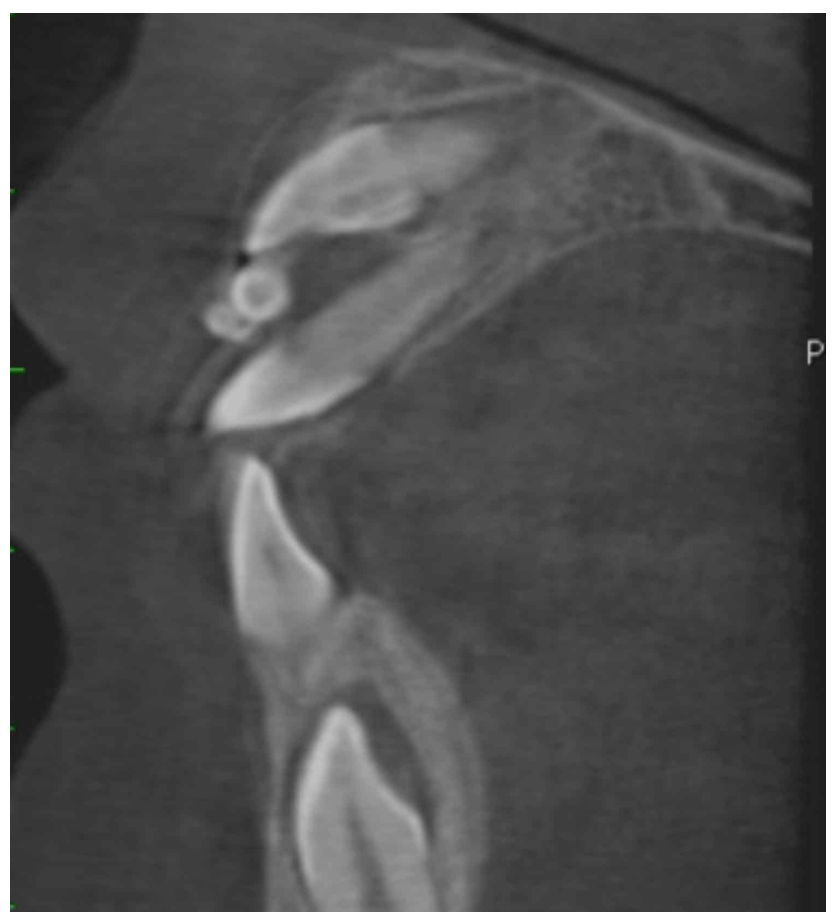

Fig. 10. Corte sagital CBCT. Diente 11 incluido, múltiples dentículos definidos en relación al diente

\section{DISCUSIÓN}

Existen múltiples casos descritos en la literatura sobre esta patología, el cual es el tumor benigno odontogénico más común. Dado su prevalencia, es necesario tener un adecuado conocimiento acerca de ellos y sus características, de manera de realizar un adecuado diagnóstico y tratamiento.

En la literatura no existe un acuerdo entre los diferentes autores acerca de la predilección del sexo del paciente. De acuerdo al meta análisis de HidalgoSánchez et al., no existen diferencias notorias en cuanto a la predilección de esta patología por el sexo del paciente. En este caso, ambos pacientes tratados fueron de sexo femenino. 
En cuanto a la edad de diagnóstico, esta es más prevalente durante la segunda década de vida, y en cuanto a la forma de diagnosticar, esta se da mayormente por medio de hallazgo radiográfico (Salgado \& Mesquita). Según el meta análisis de Hidalgo-Sánchez et al., en más de la mitad de los casos el motivo de consulta es la retención de un diente permanente, pudiendo existir también otros motivos tales como edema, persistencia de dientes temporales y agenesia de dientes permanentes. Uno de nuestros casos coincide en que la edad del diagnóstico es en la segunda década de vida, tal como la prevalencia descrita en la literatura lo informa, y el segundo caso se presenta en la primera década de vida, sin embargo, muy cercano a la segunda. Ambos casos fueron hallazgos radiográficos y el motivo de consulta principal fue la retención de diente permanente.

Respecto a la localización, existe una diferencia entre odontomas compuestos y odontomas complejos. Según la literatura, los odontomas compuestos son más comunes en la zona anterior maxilar, y los odontomas complejos más comunes en la zona posterior mandibular (Núñez et al.). Sin embargo, de acuerdo al meta análisis de Sánchez et al., de los odontomas compuestos un $59,5 \%$ fue encontrado en la zona maxilar y un $40,5 \%$ en la mandíbula. Según el reporte de nuestros casos, ambos fueron localizados en la zona maxilar, uno en la zona anterior y el otro en la zona posterior maxilar, lo que concuerda con la localización preferentemente maxilar descrita en la literatura.

Finalmente en cuanto al tratamiento, este generalmente consta de la exéresis del odontoma compuesto (Hidalgo-Sánchez et al.), pudiendo permitir así la natural erupción del diente impactadado, o bien,su tracción ortodóncica.

El reporte de Singh et al. (2015) nos muestra una opción terapéutica que consta de la exéresis del odontoma, en conjunto con el injerto óseo más PRF para preservar el hueso alveolar, y, en una segunda instancia quirúrgica, el autotransplante del diente impactado. Su evolución luego de 6 meses se muestra favorable y con buen pronóstico (Singh et al.).

En el caso $\mathrm{n}^{\circ} 1$ se realizó la exéresis del odontoma y todos sus dentículos, preservando al máximo el hueso alveolar. Se decidió la desinclusión del diente retenido y la instalación de un implante dental en conjunto con injerto óseo más PRF, todo en un mismo tiempo quirúrgico, para la posterior rehabilitación del implante.
En el caso $n^{\circ} 2$, de una paciente de 9 años de edad, se realizó la exéresis del odontoma y sus dentículos, para esperar la natural erupción del diente retenido.

\section{CONCLUSIONES}

Se puede apreciar que existen distintas medidas terapéuticas respecto a la rehabilitación de una zona edéntula producto de un odontoma compuesto, todas incluyendo su exéresis previa. Esto depende principalmente de la edad del paciente, ya que muchas veces es solo necesaria la exéresis del odontoma y esperar a la erupción natural del diente retenido. Sin embargo, cuando son requeridas medidas rehabilitadoras, para decidir entre las distintas medidas terapéuticas, un punto importante a considerar es la posibilidad de realizar un acto quirúrgico en solo tiempo operatorio para el paciente.

MARTINOVIC, G. G.; SANTORCUATO, C. B.; ALISTER, H. J. P.; PLAZA, Á. C. \& RAFFO, S. J. Compound odontoma: Diagnosis and treatment therapeutic measures. Case reports \& literature review. Int. J. Odontostomat., 11(4):425-430, 2017.

ABSTRACT: Odontomas are the most common benign odontogenic tumors and are composed of dental tissue, odontogenic epithelium and mesenchymal tissue. They are divided into two subtypes: Compound odontomas, which present normal tooth tissue, but an alteration in their conformation and size, and complex odontomas, which present well-formed tooth tissue, but are surrounded by disorganized tissue. A bibliographic review was performed by one operator in Pubmed and Epistemonikos. After filtering by title and abstract, only one systematic review was selected. We present two clinical cases of compound odontoma in female patients at the Military Hospital in Santiago. Patients were referred for evaluation and treatment due to the radiographic finding of compound odontoma. In case \# 1 , the impacted tooth was disincluded, the remaining temporal tooth was extracted, and excision of the compound odontoma carried out; subsequently a bone graft implant was placed in the remaining socket. In case \# 2, the remaining temporal tooth was extracted and compound odontoma excised. After that, natural eruption of the corresponding permanent tooth is expected. In case \# 3, tooth 1.8 fenestration and excision of compound odontoma was performed. In this case also, natural eruption of the corresponding permanent tooth is expected. Given the prevalence of this pathology, adequate knowledge of compound odontomas and their characteristics is necessary for proper diagnosis and treatment. There are 
MARTINOVIC, G. G.; SANTORCUATO, C. B.; ALISTER, H. J. P.; PLAZA, Á. C. \& RAFFO, S. J. Odontoma compuesto: Diagnóstico y tratamiento. Reporte de casos \& revisión de la literatura. Int. J. Odontostomat., 11(4):425-430, 2017.

different therapeutic measures for the rehabilitation of an edentulous area following excision of compound odontoma. This depends mainly on the age of the patient. When rehabilitation measures are required, an important aspect to consider for the patient, is the possibility of a single surgical event, as was done in case \# 1.

KEY WORDS: compound odontoma, diagnosis, therapeutic.

\section{REFERENCIAS BILIOGRÁFICAS}

Angiero, F.; Benedicenti, S.; Parker, S.; Signore, A.; Sorrenti, E.; Giacometti, E. \& Crippa, R. Clinical and surgical management of odontoma. Photomed. Laser Surg., 32(1):47-53, 2014.

Hidalgo-Sánchez, O.; Leco-Berrocal, M. I. \& MartínezGonzález, J. M. Metaanalysis of the epidemiology and clinical manifestations of odontomas. Med. Oral Patol. Oral Cir. Bucal, 13(11):E730-4, 2008.

Manterola, C. \& Astudillo, P. Checklist for reporting of descriptive observational studies. MINCIR initiative. Int. J. Morphol., 31(1):115-20, 2013.

Núñez, C. L.; Zamorano, Y. G.; Moreno, S. M.; Landaeta, M. M.; Fernández, T. M. A. \& Donoso, H. F. Odontoma complejo erupcionado: reporte de un caso. Rev. Clin. Periodoncia Implantol. Rev. Clin. Periodoncia Implantol. Rehabil. Oral, 9(1):8-12, 2016.

Salgado, H. \& Mesquita, P. Compound odontoma-Case report. Rev. Port. Estomatol. Med. Dent. Cir. Maxilofac., 54(3):161-5, 2013.

Singh, W. R.; Aheibam, K. \& Nameirakpam, A. PostOdontoma autotransplantation of an impacted tooth: $A$ case report. J. Oral Biol. Craniofac. Res., 5(2):120-3, 2015.

Thistle Barba, L.; Muela Campos, D.; Nevárez Rascón, M. M.; Ríos Barrera, V. A. \& Nevárez Rascón, A. Descriptive aspects of odontoma: literature review. Rev. Odontol. Mex., 20(4):e265-9, 2016.
Dirección para correspondencia

Gonzalo Martinovic

Cirujano Maxilofacial

Hospital Militar de Santiago

Programa Magister en Ciencias Medicas Mención Cirugía

Facultad de Medicina

Universidad de La Frontera

Temuco

CHILE

E-mail: drmartinovicg@gmail.com

Recibido : 22-09-2017

Aceptado: 14-11-2017 